\title{
Psycho-Emotional and Functional State in the Process of Intuitive Thinking
}

\author{
Tatyana Tretyak, Igor Dregval, Olena Severynovska
}

Oles Honchar Dnipropetrovsk National University, Ukraine

tessamina78@gmail.com

\begin{abstract}
Keywords: intuition, cardiovascular system, ECG, gas discharge images radiation, Kirlian method, heart rhythm variability
\end{abstract}

\begin{abstract}
The article is devoted to studying the mechanisms of regulating physiological maintenance of mental processes and the possibility of Kirlian photography for assessing psychoemotional state with intuitive thinking. While doing the tasks connected with the intuition, the students with the low level of intuitive thinking demonstrated the increase in the degree of filling blood hemoglobin with oxygen (saturation) and the increase in heart systole rate (HSR). The change of the indices of the heart rhythm variability - the mode decrease with the simultaneous increase in the mode amplitude and the vegetative indices - indicates the activation of both sympathetic and central mechanisms of mental processes regulation. The tension index of the regulatory systems (RS) allows us to judge about the mental process activity. The students were in the state of the compensated distress.

All indices of the heart rhythm variability of the students with the medium intuition level indicate the central mechanisms of regulating the intuitive thinking processes. The increase in saturation, increased RS on the background of HSR decrease in the students of this group reveals a special functional state connected with the doubled control over the processes.

The area of the Kirlian luminescence crown around the right hand forth finger is increased in all students with intuitive thinking. The energetic luminescence degree of the corresponding finger reveals the level of development of this kind of thinking: the higher the level of intuitive thinking, the more the luminescence area. Due to its high sensitivity, the Kirlian method can be applied for revealing the student's individuality that is not identified by the psychological tests.
\end{abstract}

\section{INTRODUCTION}

In creative activity, logical methods and strategies of problem-solving interact with the intuitive thinking mechanisms $[1,2]$. The most important criterion of the intuition is the fact that it is mostly revealed when the search for an answer to a difficult question discontinues, when we abandon the attempts of making rational decisions. Intuition is often connected with the instinctive guess, with gaps and leaps in the successive stages of information processing and with the presence of some unconscious experience.

R. Klexton identifies the following kinds of intuition: expertise (non-reflectory doing complicated highly-qualified actions), implicit teaching (the way of acquiring knowledge with the help of the unconscious), judgments (making an accurate decision and categorizing without being able to explain or prove it), sensitivity (increased attention to details of a situation), speculation (the process of "chewing" the experience for extracting new meanings) and creativity or using mental incubation while solving problems [3]. The presence of the incubation phase, i.e. the unconscious work over the problem and the appearance of the intuitive intention is distinguished by a number of scientists as an important constituent of the creative process [4].

The majority of the scientists studying the inner intuition come to the conclusion that the phenomenon of the intuition is based on the experience of an individual: new combinations or unusual memories application, impressions, ideas and facts that for some reasons are not accessible for the consciousness of an individual, but which are stored in the subconscious area. The harmonious interaction between the conscious and the unconscious mind plays an important role in 
the intuitive thinking mechanism. The ideas which occur unconsciously are accessible for the rational analysis only when they break into consciousness as a result of the intuitive insight, however, there is no distinct concept explaining the mechanism of this phenomenon [5].

The actuality of studying intuitive thinking is conditioned, firstly, by the significance of intuition as an important constituent of mental reality, its connection with investigating a whole number of key problems of modern psychology (creativity, thinking, problem-solving, abilities and so on). Secondly, the important role of intuition in widening cognitive abilities and actualization of the inner resources of human psychic is emphasized. Thirdly, it is conditioned by the growing practical interest in the problem of intuition as a condition of forming and a criterion of professional competence of specialists in different spheres of activity. And lastly, it is necessary for creating the artificial intelligence [6]. For studying thinking processes, both qualitative tests and quantitative methods are applied: the factor analysis in studying the intelligence structure, the correlation analysis in studying the dependence of thinking on the individual psychological characteristics of a human, the information analysis in creating artificial concepts, the methods of multi-scaling in studying emotional regulation of thinking and the methods of mathematic and programme modeling of thinking [7-9]. Each method of thinking investigation has some strong and weak points, that is why we agree with the opinion that intuitive thinking must be considered from the position of the integrative parameter of personality which is incorporated into the united subsystem of qualities of psychological and biological components [10]. To get closer to revealing the physiological mechanism of intuitive thinking, we investigated personality, psycho-emotional, functional and neurophysiologic characteristics of students engaged in cognitive activity.

Our research broadens the concepts of thinking processes presented before in various scientific investigations $[11,12]$.

\section{MATERIALS AND METHODS}

\section{Study groups}

175 girls-volunteers (aged 18-22) studying at the Faculty of Biology, Ecology and Medicine of Oles Honchar Dnipropetrovsk national university took part in the research. At this moment students didn't have any complaints of headaches, fatigue or sleepiness. Those who go in for sport and who used medication, alcohol or coffee within 24 hours before the research, were not involved in examination.

The research took place in a quiet, well-aired darkened room with the constant temperature of $+20-+22^{\circ} \mathrm{C}$, in morning hours which allowed excluding the influence of the per diem fluctuations of the vegetative heart rate (VHR) on the research results.

\section{Stages of research}

The research went through two stages.

At the first stage the individual psycho-physiological characteristics of students were studied. According to the results of the psychological testing, the right-handed, ambivert students who didn't have asthenia, possessing the medium level of stress resistance and anxiety and the strong type of nervous system, were selected. According to the type of thinking, the groups with low and medium level of intuition development were formed.

At the second stage the degree of blood hemoglobin oxygenation in the process of thinking activity was researched with the help of the finger pulse-meter of series XY300. The pulse rate and neuro-vegetative state were assessed by the variability of heart rhythm (VHR) [13].

\section{Vegetative indicators to assess the heart rate variability}

After registering 100 cycles of ECG with the help of a portable electrocardiograph EC-1 T- 03M2 and counting the duration of every cardio interval, the following indices of the cardiointerval graphics (CIG) were calculated: mode (Mo) with the formula, mode amplitude (AMo), variable amplitude (MxDMn), stress-index (SI), pNN50\% (the quality of pairs of the successive cardio intervals, distinguishing by more than $50 \mathrm{~ms}$ ) [14]. Indices pNN50 and AMo were used both 
separately and as a constituent of the complex "vegetative index" (VI), suggested by A. Ban, which was calculated by the formula:

$$
V I=\mathrm{pNN} 50 / 10+(100-A M o) / 10 \quad[15] .
$$

Before and after the mental loading, the psycho-emotional state was determined on the basis of the gas discharge image analysis (Kirlian method).

\section{Kirlian Photo Discharge method (Kirlian effect)}

Kirlian finger photography of the tested in the field of high voltage was made with the device "REK 1" designed by the Ukrainian Scientific Research Institute of Machine Building Technology (the city of Dnipropetrovsk) using the methods of L.A. Pesotskaya [16]. Registration, processing and analysis of luminescence parameters of the gas discharge image were made in the following succession: analogue- digital transformation of Kirlian images through scanning; image fragmenting with the aim of distinguishing the luminescence areas of certain fingers; image binarization by the brightness threshold; calculating the area of the luminescence crown (ALC) [17].

\section{The study of intuitive thinking}

For studying the intuitive thinking, some photos of plants, animals, mushrooms, human muscles and bones with the Latin inscriptions to them were offered. The students were to choose the right variant out of three variants offered. We should mention that all the objects were chosen from the programme material that students had learned but didn't remember by that moment. If a student knew the right answer, the question was omitted and the next one was taken.

The tested were doing the test tasks for 5 minutes in the free regime.

\section{Statistical analysis}

The data were analyzed using Microsoft Excel 2013 add STATISTICS 6.0. The analysis of variance (ANOVA) was used. The limit of statistical significance was set at $p \leq 0.05$.

\section{Results and discussions}

The success in doing the tasks connected with logical thinking was assessed by the number of the right answers that were given. On the basis of the results obtained, two experimental groups were formed: the students with the low level of intuitive thinking development (group I) who gave correct answers to $40-50 \%$ of the tasks and the students with the medium level of intuitive thinking development (group II) who gave the right answers to $75-80 \%$ of the tasks.

\section{Saturation and heart rate while intuitive thinking}

The activation of brain by the cognitive activity requires the adequate metabolic maintenance and, first of all, the intensified oxygen supply which is achieved by the blood supply intensification [18]. It was established that in a quiet state, the degree of oxygen saturation of arterial blood hemoglobin of the students with the low level of intuition development was $92.2 \%$ and it was positively increasing up to $94.5 \%$ while the students were doing the tasks connected with intuition (fig. 1). HSR in a quiet state accounted for 79.7 beats per minute, and in the process of the intuitive thinking it positively increased up to 84.8 beats per minute. The degree of oxygen saturation of arterial blood hemoglobin of the students with the high level of intuition development was (as in the previous group) $92.2 \%$ and was positively increasing with thinking activity, whereas HSR positively decreased. 
A

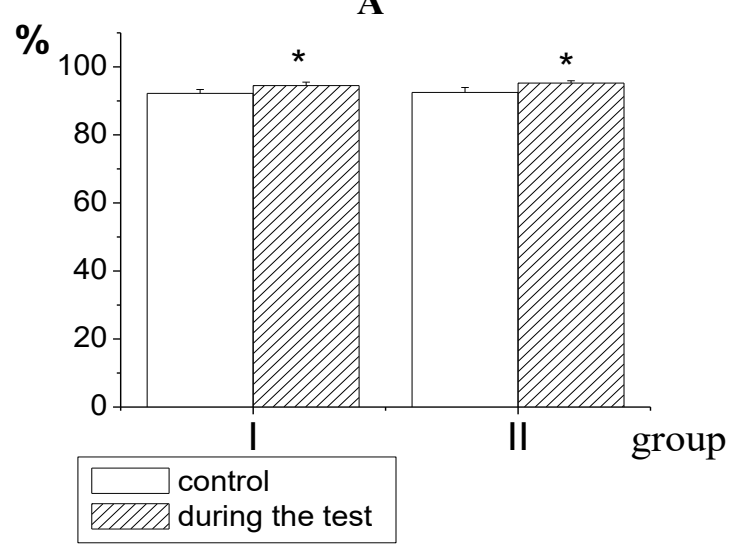

B

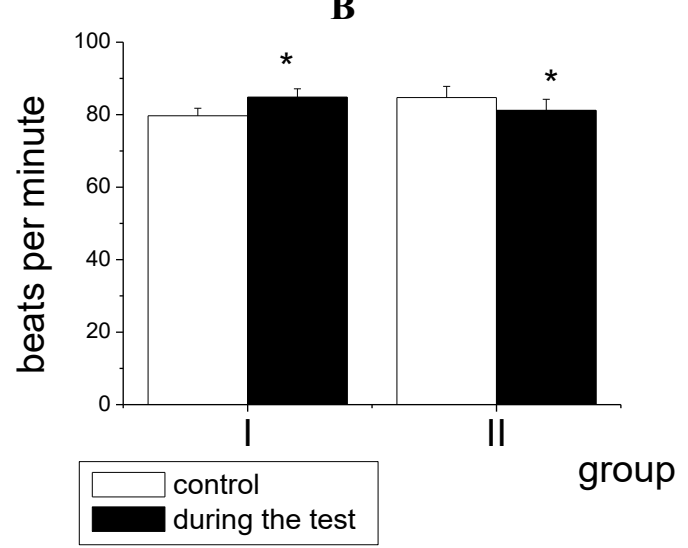

Figure 1. The degree of oxygen saturation of arterial blood hemoglobin (A) and the heart systole rate $(\mathrm{B})$ before testing (control) and in the process of intuitive thinking (during the test). $*$ - differ significantly from control $(\mathrm{p}<0.05)$.

\section{Assessment of heart rate variability with intuitive thinking}

For further studying the functional state with the cognitive activity, the calculation index of cardio interval graphics introduced by R.M. Baievsky was used. We should notice that CIG is the «golden» standard of the multi-criteria complex of methods for defining the physiological maintenance of the psychic processes by the indices of cardio-vascular system changes, commonly accepted by the scientific society. Before doing the tasks, the most frequently found cardio interval index (Mo) of ECG in students of both groups was $0.64 \mathrm{~s}$ which testifies about the prevalence of sympathetic influences (fig. 2). After the task connected with intuition, the tested from two groups demonstrated two differently directed reactions: the students of group I demonstrated the decrease in Mo (increase in sympathetic influence) while the students of group II demonstrated the increase in Mo (balancing the vegetative influences - in norm).

A
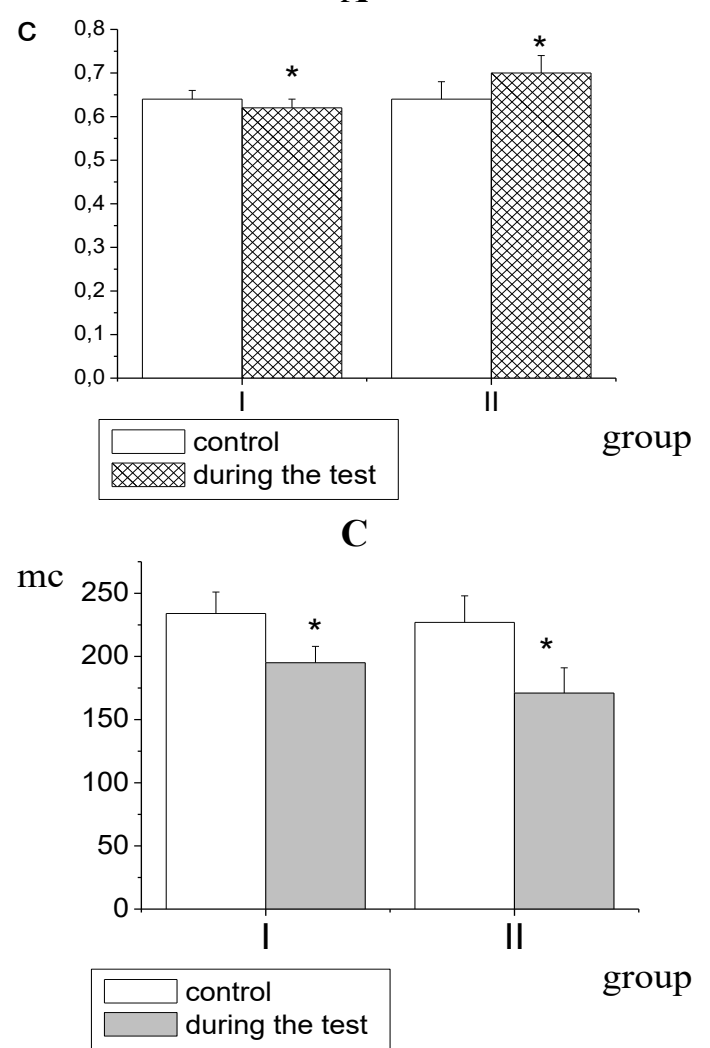

B

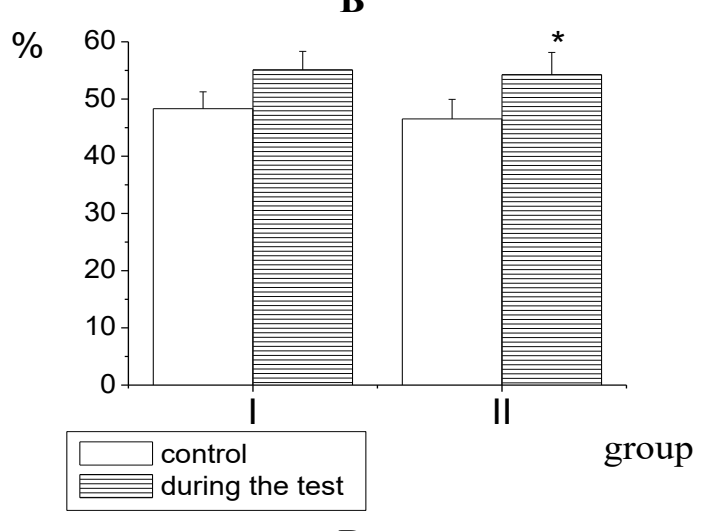

D

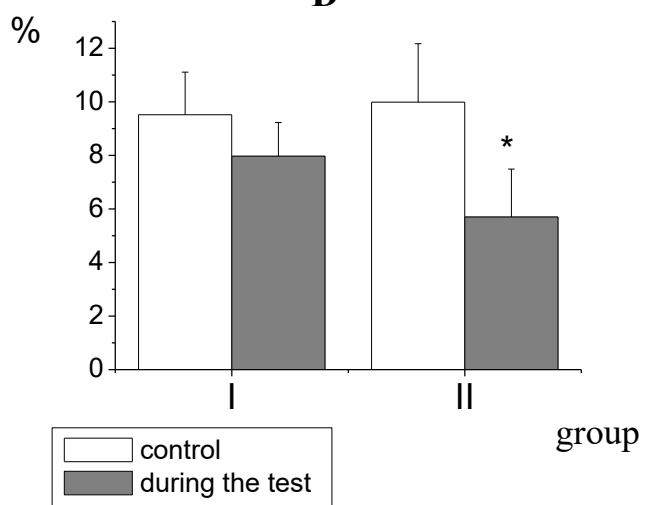

Figure 2. Indices of cardio interval graphics: mode (A), mode amplitude (b), variative span (C), pNN50 (D) before testing (control) and in the process of intuitive thinking (during the test). * - differ significantly from control $(\mathrm{p}<0.05)$. 
The mode amplitude (Amo) as a number of interval indices corresponding to Mo in per cent to the total number of cardio cycles, before the research corresponded to the higher normal limits for healthy people, whereas with mental activity it increased and exceeded $50 \%$ which testifies to the activation of the central mechanisms of psychic processes regulation, more expressed in people with the medium level of intuition abilities development.

With intuitive thinking, the variation span $(\mathrm{MxDMn})$ in the given mass of cardio cycles decreases. Actual decrease in this index in students of both experimental groups testifies to the decrease in parasympathetic influence with this kind of cognitive activity.

The decrease in index pNN50, which is more expressed in people with the developed intuition, testifies to the lack of parasympathetic influence during doing the tasks connected with intuition.

The stress index (the index of tension of regulatory systems) of the tested in the given groups exceeds the upper limit of the norm (200 conventional units) before the beginning of the research and was significantly increasing with intuition thinking (fig. 3). This testifies to the fact that the process of regulating physiological functions with cognitive activity is characterized by the prevalence of central influence reflecting the tension of system functioning and tendencies to doubling the control over the processes, especially in people with the higher indices of intuitive thinking.

A

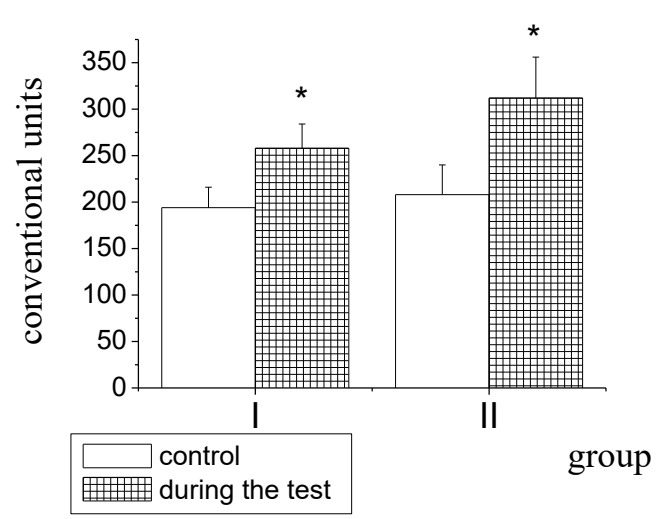

B

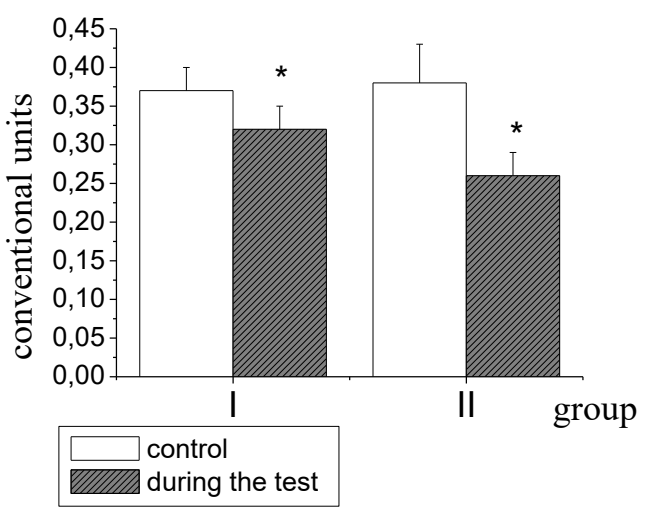

Figure 3. Indices of cardio interval graphics: stress index (A), vegetative index (B) before testing (control) and in the process of intuitive thinking (during the test). * - differ significantly from control $(\mathrm{p}<0.05)$.

At present, there is no common opinion about what index of VHR is the best for the clinical use. Artifacts, extra systoles, random phenomena and respiration rate (occasional and subjective facts) very often influence the majority of them. The fact that some of the indices are informative for reflecting the certain mechanisms of vegetative regulation is not considered to be completely proved. From these positions, indices pNN50 (reflecting the activity of the parasympathetic link of the vegetative nervous system) and AMo (characterizing the degree of centralization of the heart rhythms management) are the most suitable for assessing VHR and its dynamics. The advantages of mathematical calculation of the vegetative index (VI) are shown in the work: being complex (comprises pNN50 and AMo); relative insensitivity to random and subjective factors, simplicity of calculations for assessing the dynamics of functional state [15]. The decrease in this index with intuitive thinking testifies to the centralization of heart rhythm management, which is mostly expressed in people who are more successful while doing the tasks connected with intuitive thinking. 


\section{Evaluation of psycho-emotional state of students with intuitive thinking by the method of Kirlian}

The final stage of the research included studying the possibility of Kirlian photography for the assessment of the psycho-emotional state of a person with intuitive thinking. The basis of research of this kind was the results of the works of the researches [19]. The authors established that the images of gas discharge luminescence of human fingers contain a wide range of information signs which correlate with his psychic state. The dependence of gas discharge luminescence parameters on the psychological and physical state of a person was experimentally discovered [16].

The images registered for the fourth finger of the right hand (fig. 4), the sectors of which by P. Mandel schemes corresponded to the reactive systems (endocrine and psychic), were supposed to undergo the computer analysis [20].

A

\section{Group I}

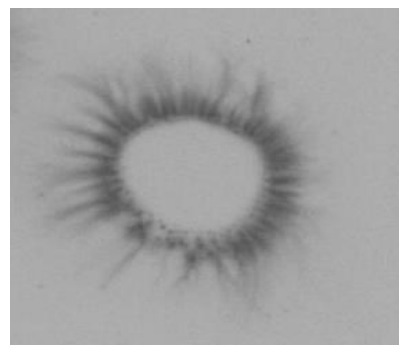

Group II

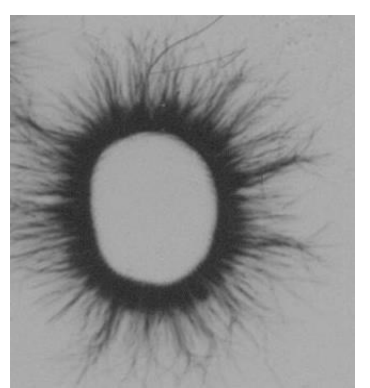

Figure 4. The examples of the images of gas discharge luminescence of the right hand fourth finger on the X-ray tape in a quiet state (A) and with the intuitive thinking (B) of students of group I and group II.

We should notice that on the basis of the analysis of gas discharge luminescence images on the X-ray tape we can obtain the data even about the insignificant signs of thinking activity, which can not be established with the help of the test assessment methods available.

The area of the luminescence crown for sure increases with intuitive thinking in students of both groups (fig. 5). The degree of luminescence energy of the corresponding finger reflects the level of development of this kind of thinking: the higher the level of intuitive thinking, the larger the luminescence area.

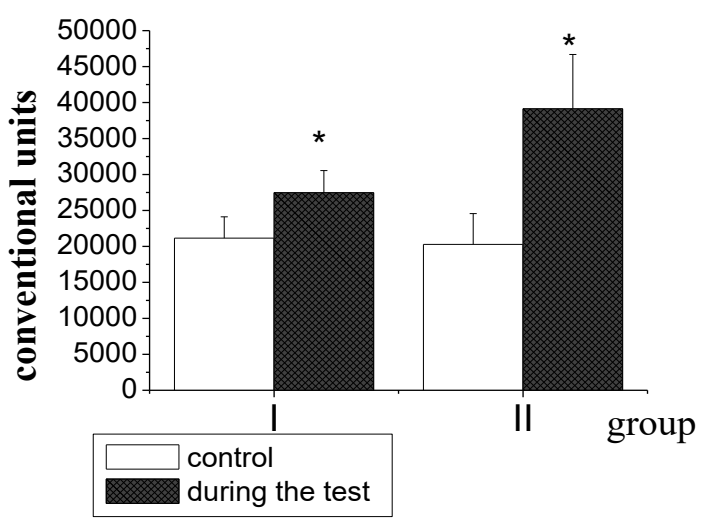

Figure 5. The area of the luminescence crown of the right hand fourth finger in a quiet state (A) and during the process of the intuitive thinking (B) of students of group I and group II. * - differ significantly from control $(\mathrm{p}<0.05)$. 


\section{Conclusions}

The results obtained show that students with the low level of intuitive thinking development (group I) while doing the tasks demonstrated the increase in oxygen supply of arterial blood (saturation) as well as the increase in HSR. It is connected with the fact that students-biologists of group I while doing the tasks connected with the intuitive choice of the right answer were quite active: they resorted to logical judgments and comparison which caused the increase in vegetative influence on the cardio-vascular system. We can judge about the sympathetic influence activation by the decrease in Mo. The increase in AMo and the decrease in the vegetative index indicate the simultaneous activation of the central mechanisms of psychic processes regulation. On the basis of the tension analysis of the regulatory systems (RS), it was established that the students were in the state of the compensated distress which testifies to the active thinking process while doing the tasks. Thus, doing the tasks by the students with the low level of the intuitive thinking was accompanied by the activation of both sympathetic influences on the heart rhythm variability and the centralization of the mechanisms of regulating physiological maintenance of psychic processes.

Students with the medium level of the intuitive thinking simultaneously demonstrated the increase in saturation and the decrease in HSR. Although the nature of intuition has not been studied sufficiently, it is known that this kind of thinking requires quietness, concentration and selfengrossment for the conscious to be able to address the subconscious and read the information. That's why people who possess intuition listen to their inner state which excludes excitement. So, although thinking is an active process, it is not supported by the activation of the sympathetic branch of the central nervous system. Indeed, all the indices of the heart rhythm variability indicate the central mechanisms of regulating the intuitive thinking processes. The increase in VI on the background of the decrease in HSR of students of group II reflects a special functional state connected with doubling the control over the processes.

The change in the area of Kirlian luminescence around the right hand fourth finger correlates with the results of the psychological tests of the assessment of the intuitive thinking development level in students. Due to its high sensitivity, the Kirlian method can be applied for revealing a student's individuality which is not identified by the results of psychological tests.

\section{References}

[1] J. Nagel, Intuition, Reflection, and the Command of Knowledge, Aristotelian Society Supplementary. 88 (1) (2014) 219-241.

[2] N.B. Novikov, About the nature of intuitive and logical components of creative thinking. Psychology faculties, Inst. of Psychology RAS, Moscow, 2005.

[3] B. Lucas, G. Claxton and E. Spencer, Progression in Student Creativity in School: First Steps Towards New Forms of Formative Assessments", OECD Education Working Papers, 86 (2013).

[4] Ut Na Sio, C.T. Ormerod, Incubation and cueing effects in problem-solving: Set aside the difficult problems but focus on the easy ones, Thinking \& Reasoning. 21 (1) (2015) 113-129.

[5] P.J. Cushen, J. Wiley, Cues to solution, restructuring patterns, and reports of insight in creative problem solving, Consciousness and Cognition, 21(3) (2012) 1166-1175.

[6] I. Hadar, When intuition and logic clash: The case of the object-oriented paradigm, Science of Computer Programming, 78 (1) (2013) 1407-1426.

[7] M.V. Malkhasyan, Cluster analysis indicators of the structure of intelligence and cognitive factors of intellectual creative children living in rural areas, Law and Life, Yurkompani, Moscow. 169 (7) (2012) 106-110.

[8] H. Ning, H. Liu, J. Mac, L.T. Yang, R. Huangc Cybermatics: Cyber-physical-social-thinking hyperspace based science and technology, Future Generation Computer Systems. 56 (2016) 504-522. 
[9] S. Atmatzidou, S. Demetriadis, Advancing students' computational thinking skills through educational robotics: A study on age and gender relevant differences, Robotics and Autonomous Systems. 75 (2016) 661-670.

[10] M.A. Cholodnaya, Psihologiya intellect: paradoxes of research, Peter, SPb ., 2002.

[11] J. Mathias, O. Josephs, J. O’Doherty, S. Zanini, B.-K. Dewar, L. Cipolotti, T. Shallice, R. J. Dolan. Human cingulate cortex and autonomic control: converging neuro-imaging and clinical evidence Brain. 126 (2003) 2139-2152.

[12] R. McCraty, M. Atkinson, and R. T. Bradley. Electrophysiological Evidence of Intuition: Part 1. The Surprising Role of the Heart. The Journal of Alternative and Complementary Medicine. 10(1) (2004) 133-143.

[13] R.M. Baevsky, A.P.Bersenieva, The analysis of heart rate variability using different electrocardiographic systems method. Recommendations, Moscow, 2002.

[14] I.I. Eliseeva, M.M. Yuzbashev, General Theory of Statistics. Finance and Statistics, Moscow, 2002.

[15] A.S. Ban, G.M. Zagorodny, Vegetative index for evaluating variability heart rate athletes, Medical Journal. 4 (2010) 127-130.

[16] L.A. Pesotskaya, The method of rapid diagnosis of mental and emotional state of a person, Patent of Ukraine №49283 A61B5/05, Bul 9 (2002).

[17] L.A. Pesotskaya, N.V. Glukhova, Development of a method of analysis of geometric and brightness parameters of the gas-discharge light images, Conf. "Biophysical Methods in Clinical Laboratory Diagnostics", St. Petersburg, 2015.

[18] L.D. Korovina, T.M. Zaporozhets, Relations between blood supply of brain of students and condition of autonomic nervous system and risk factors, Visnyk of Dnipropetrovsk Univ. Biology, medicine. 6 (1) (2015) 68-73.

[19] T.V. Popova, O.S. Kolosov, I. E. Bulatov, Gas discharge visualization method in the study of physical and mental status of the person, Bulletin of South-Ural. State. Univ. 28 (2012) 40-45.

[20] P. Mandel, Energetishe Terminalpunkt - Diagnos, FRG, ESSEN, 1983. 